\title{
All-Flavor Searches for Dark Matter with the IceCube Neutrino Observatory
}

\author{
The IceCube Collaboration ${ }^{\dagger}$, \\ ${ }^{\dagger}$ http://icecube.wisc.edu/collaboration/authors/icrc15_icecube \\ E-mail: klaus.wiebe@uni-mainz.de
}

Dark matter particles can be trapped in massive celestial bodies, such as the Sun or the Earth and their self-annihilations may produce standard model particles, including neutrinos of all flavors. So far, IceCube dark matter searches have focused on muon neutrinos due to their track-like topology and the resulting good pointing resolution. However, recent developments of reconstruction tools have allowed us to reconstruct electron and tau neutrino interactions with sufficiently good angle and energy resolutions and to estimate the corresponding uncertainties. IceCube's in-fill array DeepCore, when using the outer IceCube detector as a veto, permits us to extend all-flavor dark matter searches to energies well below neutrino energies of $100 \mathrm{GeV}$. This is particularly important for the search of Weakly Interacting Massive Particles (WIMPs) that accumulate in the center of the Earth, as their annihilation rate is expected to be enhanced for WIMP masses around $50 \mathrm{GeV} / \mathrm{c}^{2}$. All-flavor neutrino searches, in principle, enhance IceCube's sensitivity with respect to previous searches based solely on muon neutrinos. While this paper primarily focuses on demonstrating the applied methods, we will also present a sensitivity and discovery potential for an Earth WIMP search as well as data selection efficiencies for an ongoing solar analysis. We find that efficient neutrino flavor identification is challenging at low energies given that the signatures for tracks and showers are very similar. For the proposed event selection the signal rate increases by a factor of two. However, the worse angular resolution for cascades gives rise to a larger background in the signal region.

Corresponding authors: Klaus Wiebe*1, Anna Steuer ${ }^{1}$

${ }^{1}$ Institute of Physics, University of Mainz, Germany

The 34th International Cosmic Ray Conference,

30 July- 6 August, 2015

The Hague, The Netherlands

${ }^{*}$ Speaker. 


\section{Introduction}

The IceCube Neutrino Observatory, located at the geographic South Pole, consists of the IceCube neutrino telescope and the IceTop air shower array [1]. In the ice, a volume of one cubic kilometer is instrumented with 5160 digital optical modules (DOMs), deployed at depths between $1450 \mathrm{~m}$ and $2450 \mathrm{~m}$. The denser low energy infill, DeepCore, considerably improves neutrino detection with energies below $100 \mathrm{GeV}$ due to the higher sensor granularity and the veto capacity of the surrounding IceCube strings.

IceCube can detect all flavors of active neutrinos through Cherenkov light emission from secondary particles created when a neutrino interacts in the ice. The primary background in the search for neutrinos originates from cosmic ray hadronic air showers produced in the Earth's upper atmosphere. The decay of pions, kaons (and charmed mesons) results in a continuous stream of neutrinos and penetrating muons. High energy muons are capable of traveling long distances through matter before they eventually decay, resulting in a downgoing muon flux at the IceCube detector. In contrast to earlier solar analyses we do not restrict ourselves to periods where the Sun is below the horizon and the Earth forms a shield against cosmic ray muons. Instead we take advantage of IceCube's veto capabilities, which limits the accessible WIMP mass range below $1 \mathrm{TeV} / \mathrm{c}^{2}$.

Muon neutrinos with extended track-like topologies are relatively easy to reconstruct with degree pointing precision. The reconstruction of electron and tau neutrino interactions, leaving cascade-like signatures in the detector, is more challenging. Due to this better angular resolution, Earth WIMP searches [2] as well as solar WIMP searches [3] with IceCube have until now aimed at extracting solely $v_{\mu}$ events from the dataset. However, all-neutrino searches have become more important recently. The reasons are obvious [4]: the measured flux is enhanced, the neutrino energies may be determined to a better precision, backgrounds from atmospheric $v_{\mathrm{e}}$ and $v_{\tau}$ are smaller and cosmic ray muons tend to be rejected better by requiring that the events have a cascadelike signature.

This paper discusses a study of IceCube's sensitivity to WIMP annihilations in the centers of the Earth and Sun with an analysis that is sensitive to all flavors of neutrinos. The methods presented in sections 3 and 4 have been developed for application on one year of 86-string configuration IceCube data from the 2011 season. Note that while both Earth and Sun may trap WIMPs, only the Sun is expected to be in an equilibrium making the annihilation rate directly proportional to the WIMP-Nucleon scattering cross section. The lower mass of the Earth prohibits this equilibrium in most cases. Similar to the moderation process in nuclear reactors, WIMPs are most efficiently slowed down if their energy is in the mass range of their nuclear scattering partners. As iron is very abundant in the Earth's core, $50 \mathrm{GeV} / \mathrm{c}^{2}$ mass WIMPs are most easily captured, thereby enhancing the annihilation rate. For the simulation of a WIMP-induced neutrino signal we use the WimpSIM package [5], which takes care of neutrino generation, propagation and oscillations.

\section{Low Energy Cascade Reconstruction}

Unlike the extended tracks caused by muons from charged-current (CC) $v_{\mu}$ events, $v_{\mathrm{e}}$ and $v_{\tau}$ leave an almost spherical pattern of hit DOMs in the detector. The $\mathrm{e}^{ \pm}$produced in CC $v_{\mathrm{e}}$ interactions are subject to successive bremsstrahlung energy losses and lead to electromagnetic cascades. 
$\nu_{\tau}$ interactions and $\tau$ decays predominantly produce hadronic cascades, as do neutral-current interactions from all neutrino flavors. While the energy reconstruction benefits from the confined event signature, a good directional reconstruction of the spherically shaped cascade events demands significant computing resources and also an excellent description of the ice properties [6]. Energy $E$, position and orientation are estimated [7] by minimizing the negative log-likelihood $-\ln L=\sum_{i} k_{i} \ln \left(E \Lambda_{i}+\rho_{i}\right)-\left(E \Lambda_{i}-\rho_{i}\right)-\ln k_{i}$ !, where $\rho_{i}$ is the expected number of noise photons. The number of photons per unit energy for an assumed orientation and vertex $\Lambda_{i}$ incorporates detailed information on the position dependent absorption and scattering of photons in the ice. This information is available in the form of spline-fitted [8] tables obtained from a photon-tracking simulation using a ray tracing algorithm modeling scattering and absorption. When iterating the minimization chain (in this analysis 32 times) and optimizing minimization parameters, the resulting angular resolutions are similar to the ones seeded by the true direction and vertex.

The energy dependent median spatial angle resolution is shown in Fig. 1. The analyses presented in the following sections focus on rather low energies where the discussed methods are competitive, but an efficient particle identification of cascades and tracks is not possible. Therefore all flavors - including muon (see Fig. 1, solid orange line) - are first reconstructed with a cascade event hypothesis.

Individual event resolutions may vary from the average resolution (see Fig. 1) dependent on the event's exact topology and the amount of light deposited in the detector volume. Since event-based resolutions allow for a reconstruction quality based event weighting, a

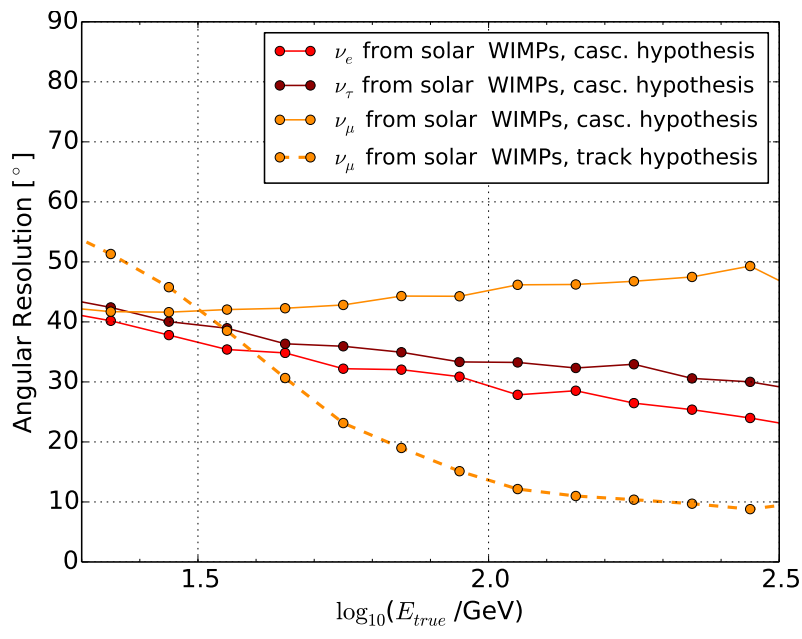

Figure 1: Angular resolution of the cascade reconstruction algorithm versus the energy of neutrinos. The resolution improves with energy for cascade signatures. For comparison, the dashed orange line shows the resolution potential for tracks reconstructed by an algorithm using the correct track hypothesis. resolution estimator, based on the Cramer-Rao upper bound on the variance, was coded. Assuming a set of parameters $\vec{\theta}=\left(x_{0}, y_{0}, z_{0}, \theta, \phi\right)$, the vertex and directional angles, we formulate a Poissonian likelihood ${ }^{1}$

$$
L(\vec{\theta})=\prod_{i=1}^{\# \text { \#hitDOMs }}\left(\prod_{j=1}^{\text {nbins }(i)} \frac{\mu_{\mathrm{h}}(\vec{\theta}, i, j)^{n(i, j)}}{n(i, j) !} \cdot \exp \left(-\mu_{\mathrm{h}}(\vec{\theta}, i, j)\right)\right) \cdot \prod_{k=1}^{\# \text { nonhitDOMs }} \exp \left(-\mu_{\mathrm{nh}}(\vec{\theta}, k)\right)(2.1)
$$

with $\mu_{\mathrm{h}}$ being the expected number of photons in module $i$ for time bin $j$ ( $\mu_{\mathrm{nh}}$ for a non-hit module $k$ respectively) and $n$ the actually measured number of photons.

In order to obtain resolution expectations for individual cascades, one can either scan the likelihood around its minimum or take advantage of the Cramer-Rao bound. Under certain conditions,

\footnotetext{
${ }^{1}$ Similar to the likelihood formulated in the beginning of the section, but without explicit energy formulation and neglecting noise.
} 
the latter provides the relation $\left(\operatorname{cov}^{-1}\right)_{l m}=F_{l m}=-\left\langle\frac{\partial^{2} \log L(\vec{\theta})}{\partial \theta_{l} \partial \theta_{m}}\right\rangle$, where $F$ is the Fisher Information matrix, the inverse of the covariance matrix. Applying the second derivative and exploiting $\langle n(i, j)\rangle=\mu_{h}(\vec{\theta}, i, j)$ we obtain:

$$
F_{l m}=\sum_{i=1}^{\# \text { \#hitDOMs nbins }(i)} \sum_{j=1}^{\frac{1}{\mu_{\mathrm{h}}(\vec{\theta}, i, j)}} \frac{\partial \mu_{\mathrm{h}}(\vec{\theta}, i, j)}{\partial \theta_{l}} \frac{\partial \mu_{\mathrm{h}}(\vec{\theta}, i, j)}{\partial \theta_{m}}+\sum_{k=1}^{\# \text { \#onhitDOMs }} \frac{\partial^{2} \mu_{\mathrm{nh}}(\vec{\theta}, k)}{\partial \theta_{l} \partial \theta_{m}} .
$$

The expected value for the number of registered photons for DOM $i$ and time bin $j, \mu_{\mathrm{h}}(\vec{\theta}, i, j)$, is obtained from the spline fitted tables discussed above. In order to accelerate the algorithm, optical modules not hit and the actual amount of detected photons as well as their timing information are currently ignored. Standard deviations calculated from the diagonal entries of the covariance matrix correlate well with the actual resolutions. The estimate on the spatial resolution is approximated by $\sigma_{\text {spatial }}=$ $\sqrt{\sigma_{\theta}^{2}+\left(\sigma_{\varphi} \cdot \sin \theta_{\text {reco }}\right)^{2}}$, where $\sigma_{\theta / \varphi}$ are the zenith and azimuth uncertainties and

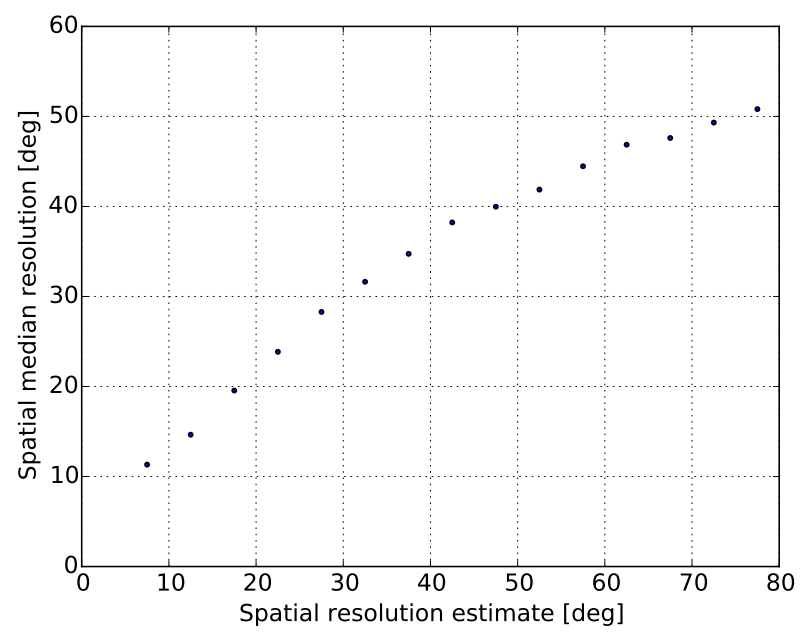

Figure 2: Cascade Resolution Estimator: a successful quantitative modeling of the actually achieved resolution.

$\theta_{\text {reco }}$ is the reconstructed zenith angle. Figure 2 shows the relation between the median resolution taken from the difference of reconstructed and true event direction and the estimate.

\section{Search for dark matter annihilation in the center of the Earth}

As a test case, we restrict ourselves in this study to $50 \mathrm{GeV} / \mathrm{c}^{2}$ WIMPs. This has the advantage that we can make use of the enhanced cross section. The disadvantage is that neutrinos from the annihilation are very low in energy and thus are at the threshold of detection. The most energetic neutrinos at these energies stem from the annihilation in tau pairs and for this reason we concentrate first on this channel. In order to extract this signal from the dataset, an event selection favoring potentially well reconstructed, low energetic neutrino events mainly from the northern hemisphere was developed. For example, the reconstructed energy was required to

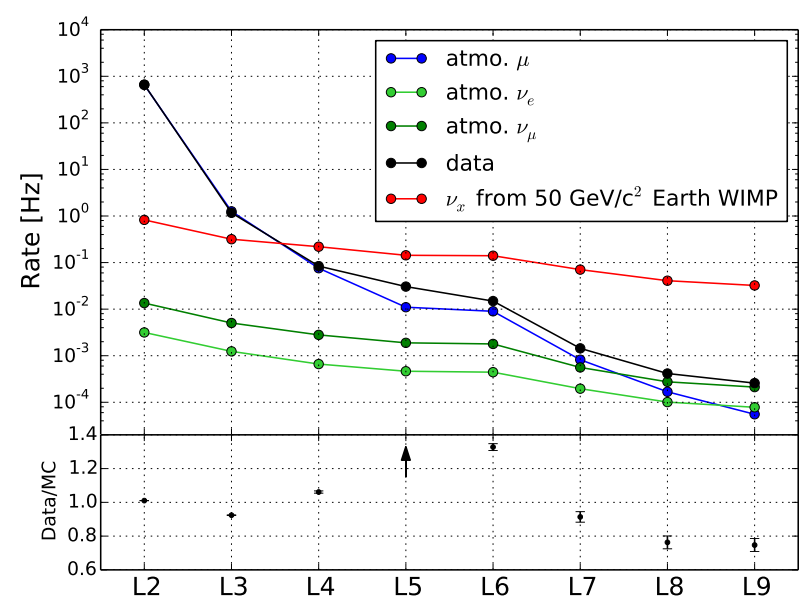

Figure 3: Rates of signal (arbitrarily scaled for comparison), background MC and data versus selection levels. The lower subplot shows the ratio of data over the sum of the background MC rates and its statistical uncertainty. The arrow indicates an outlier not visible at the scale chosen. 
be in the 5 to $30 \mathrm{GeV}$ range. This selection is subdivided into levels, each combining requirements pursuing a similar objective.

The progress with the selection levels of signal and background rates from Monte Carlo (MC) simulations and the data rates is shown in Fig. 3. Data here refers to a subset of the full experimental dataset ${ }^{2}$. The applied event selection achieves a signal efficiency of about $4 \%$ while the atmospheric muon background is reduced by seven orders of magnitude. As can be seen in the lower portion of Fig. 3, showing the data-MC-agreement, the data rate falls short of the MC rate by about $25 \%$ on the final selection level. Outliers on intermediate levels can mostly be explained by unsimulated noise. This discrepancy is removed by subsequent noise-rejecting vetos. The precise MC description of experimental data in the low energy regime is a non-trivial issue which is currently subject to collaboration-wide investigations.

Since the event simulation and the simulation of the detector response near the detector's energy threshold is challenging, the method to analyze a potential WIMP signal should not rely on precise MC background predictions. A further difficulty is the particle identification at very low energies as well as the directional reconstruction of low energy events in general and cascades in particular. A likelihood fitting procedure takes advantage of distinguishing features of cascade and track signatures and the angular distribution of signal and backgrounds. The basic input for the algorithm are two-dimensional histograms of two reconstructed zenith angles - one developed to reconstruct cascade-like events the other well suited for the reconstruction of tracks. For the case of signal events coming from the direction of the Earth's core, one would e.g. expect the cascade likelihood to recover the direction of the cascade-like events while the algorithm using the incorrect track hypothesis smears this directional peak. This concept also reveals distributional differences between atmospheric electron and muon neutrinos and discriminates the atmospheric muon background. In order to pass this distributional information efficiently to the fitter, the twodimensional histograms are binned such that signal regions are well resolved while the background dominated parts are merged. For the sake of simplicity and in order to increase the MC statistics, the three signal channels are combined into one all-flavor flux $\chi$. Fig. 4 shows a comparison of the histograms of WIMP-induced neutrinos and atmospheric neutrinos and demonstrates the discrimination potential of this method. The histogram for experimental data is analyzed by an algorithm maximizing the Poissonian likelihood

$$
L=\prod_{\text {bins }} \frac{\lambda_{i}^{k_{i}}}{k_{i} !} e^{-\lambda_{i}}+\text { nuisance terms },
$$

with the MC prediction

$$
\lambda_{\mathrm{i}}=\gamma \cdot \lambda_{\mathrm{i}}^{\text {atmo. } \mu}+\Delta \mathrm{r}_{\pi / \mathrm{K}, v_{\mu}} \cdot \mu \cdot \lambda_{\mathrm{i}}^{\text {atmo. } v_{\mu}}+\Delta \mathrm{r}_{\pi / \mathrm{K}, v_{\mathrm{e}}} \cdot \varepsilon \cdot \lambda_{\mathrm{i}}^{\text {atmo. } v_{\mathrm{e}}}+\alpha \cdot \lambda_{\mathrm{i}}^{\chi}
$$

and data $k_{i}$ in bin $i$ and the physical fit parameter $\alpha$ revealing which annihilation rate is compatible with the experimental data. Nuisance parameters included in this likelihood account for the relevant systematic uncertainties. These are the absolute flux normalizations of the atmospheric backgrounds and the pion to kaon ratio in the generation of atmospheric neutrinos which is contained in the weight $\Delta r_{\pi / K, v_{\ell}}$ in Eq. 3.2. Thus, the final log-likelihood function, including Gaussian

\footnotetext{
${ }^{2}$ In IceCube, selection criteria are developed on typically $10 \%$ of the experimental data to avoid biased results.
} 
penalty factors for the nuisance parameters, reads:

$$
-\ln L=\sum_{\text {bins }} \lambda_{i}-k \cdot \ln \lambda_{i}+\frac{1}{2} \cdot\left(\frac{(\gamma-1)^{2}}{\sigma_{\gamma}^{2}}+\frac{(\mu-1)^{2}}{\sigma_{\mu}^{2}}+\frac{(\varepsilon-1)^{2}}{\sigma_{\varepsilon}^{2}}+\frac{(r-1)^{2}}{\sigma_{r}^{2}}\right) .
$$

The incorporated nuisance parameters together with their priors and uncertainties are summarized in Table 1.

\begin{tabular}{|c||c|c|c|}
\hline uncertainty & nuisance parameter $\mathrm{n}$ & default value & $\sigma_{n}$ \\
\hline \hline normalization of atmospheric $\mu$ & $\gamma$ & 1.0 & 0.3 \\
\hline normalization of atmospheric $v_{\mu}$ & $\mu$ & 1.0 & 0.3 \\
\hline normalization of atmospheric $v_{\mathrm{e}}$ & $\varepsilon$ & 1.0 & 0.3 \\
\hline pion-kaon ratio & $r$ & 1.0 & 0.1 \\
\hline
\end{tabular}

Table 1: Summary of the nuisance parameters explicitly implemented in the likelihood function together with their priors and uncertainties.
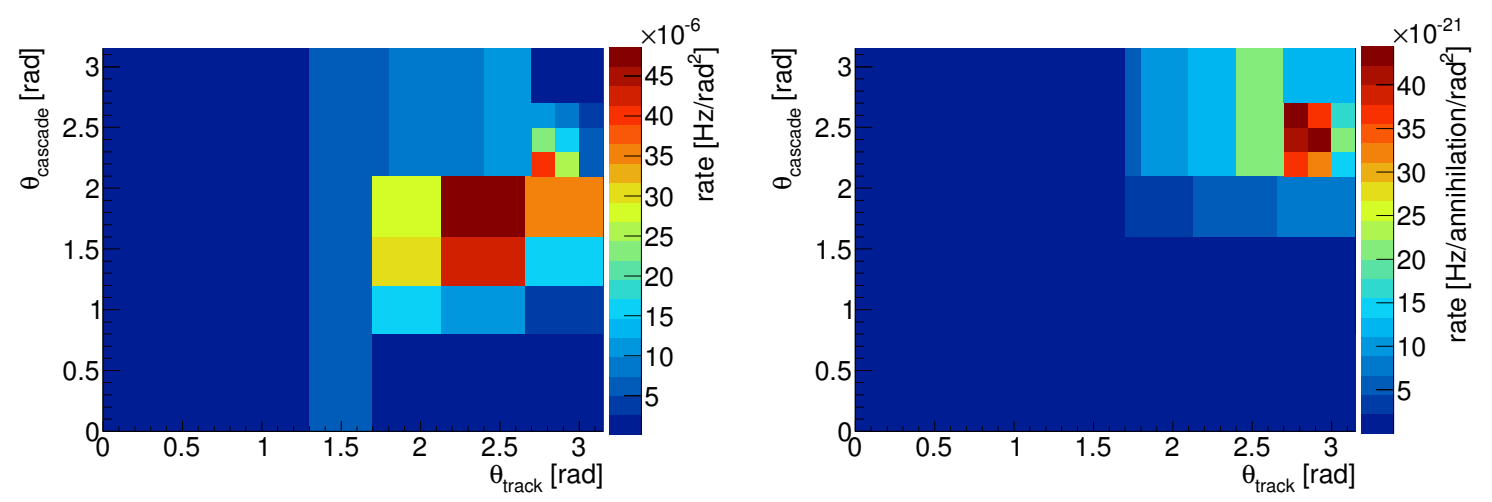

Figure 4: Two-dimensional histogram of reconstructed zenith angles with apt binning for atmospheric electron neutrinos (left) and for WIMP induced neutrinos (right). The bin contents are normalized to the bin size to emphasize the relative contributions.

To determine the sensitivity, evidence and discovery potential, a likelihood-ratio test is performed on simulated data using the test statistic $w=2 \cdot \ln \left(\frac{\max ^{2} L}{\max _{H_{0}} L}\right)$, where $\max _{H_{0}} L$ denotes the maximum likelihood under the null hypothesis while $\max L$ refers to the maximum likelihood under the alternative, i.e. signal, hypothesis. From the comparison of the test statistic distribution for many simulated data realizations containing a certain signal strength with simulated background only data realizations the sensitivity, evidence and discovery potential were calculated for one year of IceCube data (see Table 2).

Since the Earth is not expected to have reached equilibrium of WIMP capture and annihilation, the annihilation rate is not directly correlated with the WIMP-nucleon scattering cross section. For this reason, the sensitivities are given in terms of annihilation rates which maintains as much model independence as possible.

The sensitivity determined in this analysis is not competitive yet with a dedicated study for Earth WIMPs solely based on $v_{\mu}$ events [2]. The implementation of multivariate techniques promises the required enhancement of the efficiency, as can be seen in the following section. 


\begin{tabular}{|c||c|}
\hline & annihilation rate [annihilations/s] \\
\hline \hline Sensitivity $(90 \%$ Confidence Level) & $7.7 \cdot 10^{13}$ \\
\hline Evidence $(3 \sigma)$ & $1.7 \cdot 10^{14}$ \\
\hline Discovery Potential $(5 \sigma)$ & $2.2 \cdot 10^{14}$ \\
\hline
\end{tabular}

Table 2: Sensitivity, evidence and discovery potential for one year of IceCube data taken with its 86-string configuration assuming a $50 \mathrm{GeV} / \mathrm{c}^{2}$ mass WIMP annihilating into $\tau^{+} \tau^{-}$.

\section{Search for dark matter annihilation in the center of the Sun}

The indirect detection of solar WIMP particles with IceCube places some of the most stringent limits on the spin-dependent nucleon-WIMP scattering cross-section [9, 10]. Here we are exploring the capabilities of the all-flavor approach, by taking advantage of the cascade reconstruction methods discussed in section 2 .

Figure 5 shows the development of data and $\mathrm{MC}$ rates throughout the event selection. For the signal MC, a showcase WIMP candidate mass of $100 \mathrm{GeV} / \mathrm{c}^{2}$ is chosen, while the complete analysis will cover a candidate mass range from 50 to $1000 \mathrm{GeV} / \mathrm{c}^{2}$. Cut levels 3 and 4 aim at significantly reducing the dominant background from atmospheric muons, followed by filters that effectively remove noise clusters and coincident events.

Similar to the Earth WIMP analysis, we see differences between data and Monte Carlo which we suspect to be due to a non-perfect description of the optical module noise for the particular time period. Concerted efforts are under way in the collaboration to remedy this situation.

\begin{tabular}{|c||c|c|}
\hline $\begin{array}{c}\text { Candidate mass } \\
{\left[\mathrm{GeV} / \mathrm{c}^{2}\right]}\end{array}$ & $\begin{array}{c}\text { Hard channel efficiency } \\
\left(\mathrm{W}^{+} \mathrm{W}^{-}, \tau^{+} \tau^{-} \text {for } 50 \mathrm{GeV} / \mathrm{c}^{2} \text { WIMPs }\right)[\%]\end{array}$ & $\begin{array}{c}\text { Soft channel efficiency } \\
(\mathrm{b} \overline{\mathrm{b}})[\%]\end{array}$ \\
\hline \hline 50 & $8.6\left(v_{e}: 7.5, v_{\mu}: 9.7, v_{\tau}: 7.5\right)$ & $12.5\left(v_{e}: 10.5, v_{\mu}: 14.4, v_{\tau}: 9.7\right)$ \\
\hline 100 & $5.0\left(v_{e}: 4.5, v_{\mu}: 5.7, v_{\tau}: 4.5\right)$ & $8.4\left(v_{e}: 7.2, v_{\mu}: 9.7, v_{\tau}: 7.0\right)$ \\
\hline
\end{tabular}

Table 3: Selection efficiencies

Following level 5, a set of Boosted Decision Trees (BDTs) is trained to discriminate signallike from background-like events, leading to level 6 by selecting events with a BDT score larger than 0.04 (see Fig. 6). Twelve variables are used as BDT input, including reconstructed direction, energy and vertex, reconstruction quality parameters as well as veto and geometrical quantities. An overtraining check was performed, showing good agreement of training and testing score distributions. The efficiency at level 6 , compared to level 2 , is $\approx 10^{-6}$ for atmospheric muons and $5.0 \%$ 
(8.4\%) for signal neutrinos from the $\mathrm{W}^{+} \mathrm{W}^{-}(\mathrm{b} \overline{\mathrm{b}})$ channel. Signal efficiencies are shown in Table 3 and are competitive to the final-level efficiencies obtained with a track-based approach [10]. Ultimately, we want to include WIMP masses up to $1 \mathrm{TeV} / \mathrm{c}^{2}$, but concentrate here on the low energetic 50 and $100 \mathrm{GeV} / \mathrm{c}^{2}$ candidates.

For the determination of limits we will choose a likelihood approach [11] that will consider direction, directional uncertainty estimates as well as the reconstructed energy.

\section{Conclusions}

For the first time, an all-flavor analysis for WIMP searches has been made possible by the development of better angular reconstruction techniques for cascades at low neutrino energies. In order to limit the background from atmospheric muons, the analysis was restricted to events reconstructed in the volume of the DeepCore sub-array by exploiting the veto-capacity of the surrounding IceCube sensors. To maximize the selection efficiency, no flavor-separation - which is very difficult to achieve at low energies was attempted. In the future, a combination of the different analysis strategies, especially for smaller candidate masses, should be considered.

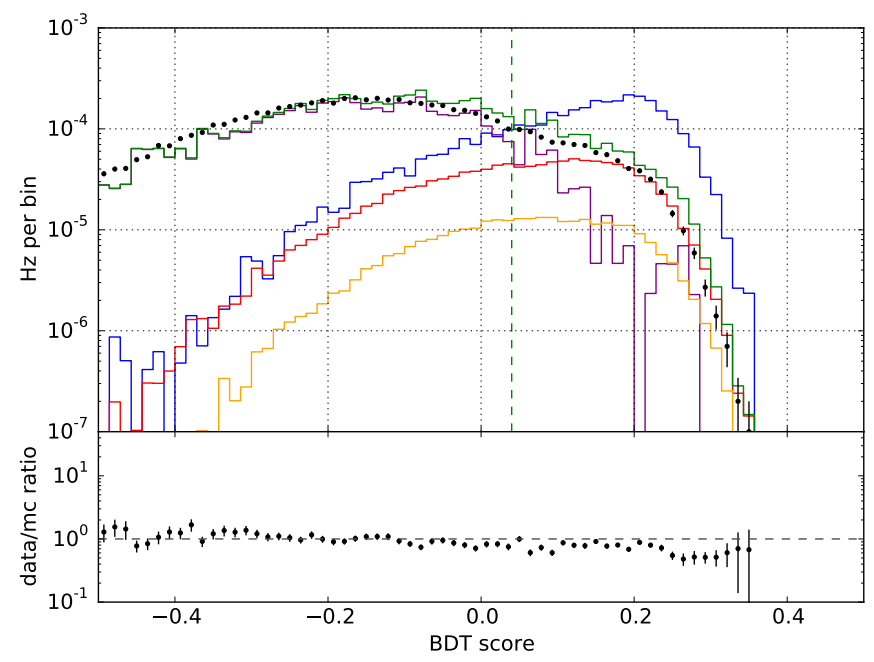

Figure 6: Score distribution after BDT training. Events with a spatial angle deviation from the solar position larger than 40 degrees are defined as "off-source". Such data (black) are used to train against the signal simulation (blue, shown here for a $100 \mathrm{GeV} / \mathrm{c}^{2}$ candidate). We reserve $50 \%$ of the data and the Monte Carlo signal events for overtraining checks. Shown for comparison are atmospheric muons (purple) and $v_{\mu}\left(v_{e}\right)$ in red (orange). The total background MC rate is depicted in green. The vertical dashed line shows the applied cut value at a BDT score of 0.04 .

\section{References}

[1] The IceCube Collaboration: A. Achterberg et al., Astropart.Phys. 26 (2006) 155 [arxiv:astro-ph/0604450]

[2] IceCube Coll., A search for Dark Matter in the centre of the Earth with the IceCube neutrino detector, PoS(ICRC2015)1205, these proceedings

[3] The IceCube Collaboration: M.G. Aartsen et al., Phys. Rev. Lett. 110 (2013) 131302 [arxiv:1212.4097]

[4] C. Rott, T. Tanaka and Y. Itow, JCAP 1109 (2011) 029 [arxiv:1107.3182]

[5] M. Blennow, J. Edsjö and T. Ohlsson, JCAP 0801 (2008) 021 [arXiv : 0709.3898]

[6] The IceCube Collaboration: M.G. Aartsen et al., Nucl.Instrum.Meth. A711 (2013) 73-89 [arXiv:1301.5361]

[7] The IceCube Collaboration: M.G. Aartsen et al., JINST 9 (2014) P03009 [arXiv:1311.4767]

[8] N. Whitehorn et al., Comput.Phys.Commun. 184 (2013) 2214-2220 [arxiv:1301.2184]

[9] IceCube Coll., Search for Dark Matter annihilations in the Sun using the completed IceCube neutrino telescope, PoS(ICRC2015)1209, these proceedings

[10] IceCube Coll., Improved methods for solar Dark Matter searches with the IceCube neutrino telescope, PoS(ICRC2015)1099, these proceedings

[11] IceCube Coll., Results of neutrino point source searches with 2008-2014 IceCube data above $10 \mathrm{TeV}$, PoS(ICRC2015)1047, these proceedings 